Scientific journal

PHYSICAL AND MATHEMATICAL EDUCATION

Has been issued since 2013.

Науковий журнал

ФІЗИКО-МАТЕМАТИЧНА ОСВІТА

Видається з 2013.
ISSN 2413-158X (online)

ISSN 2413-1571 (print)

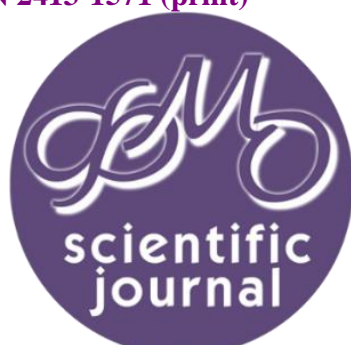

Бакиров Т.Ю. Об изучении темы "Комплексные числа» в общеобразовательной школе и в вУЗах Республики Узбекистан. Фізико-математична освіта, 2021. Випуск 5(31). С. 17-22.

Bakirov T. The continuity of the study on thetopic "Complex numbers" in secondary schools and in pedagogical universities of the Republic of Uzbekistan. Physical and Mathematical Education, 2021. Issue 5(31). P. 17-22.

DOI 10.31110/2413-1571-2021-031-5-003

Удк 51(077)+511.24

Т.Ю. Бакиров

Ферганский государственный университет, Узбекистан bakirovt75@mail.ru https://orcid.org/0000-0001-9848-9542

\title{
ОБ ИЗУЧЕНИИ ТЕМЫ «КОМПЛЕКСНЫЕ ЧИСЛА» В ОБЩЕОБРАЗОВАТЕЛЬНОЙ ШКОЛЕ И В ВУЗАХ РЕСПУБЛИКИ УЗБЕКИСТАН
}

АННОТАЦИЯ

Формулировка проблемы. В связи с реформами, проводимыми в сфере образования в Республике Узбекистан, меняется содержание среднего образования, в частности, математического образования. В этой статье рассматривается вопрос преемственности изучения темы “Комплексные числа» в школе и педагогическом вУзе, и изменение содержания среднего образования, в частности, математического образования. В программу школьной математики Республики Узбекистан были введены такие новые разделы как комбинаторика, элементы математической логики, комплексные числа, элементы теории вероятности, элементы математической статистики и финансовой математики. В результате некоторые темы или их содержание повторяются и в курсе вузовской математики. Необходима разработка методики изучения вновь вводимых разделов математики, в особенности, некоторых повторяющихся тем, определить уровни усвоения повторяющихся понятий учениками школ и студентами вузов.

Материалы и методы. В ходе исследования были применены различные методы исследования, а именно:изучение и анализ научно-педагогической, методической, математической литературы и школьных учебников по математике; анализ преемственных связей раздела «Комплексные числа»; педагогический эксперимент с целью проверки остаточных знаний учащихся по теме "Комплексные числа», в котором участвовали 89 студентов первого курса Ферганского государственного университета; обсуждение материалов исследования.

Результаты. В ходе исследования былипредложены уровни усвоения учебных элементов и ступеней абстракции, характеризующих язык изложения учебной информации по теме «Комплексные числа».

Выводы. Полученные результаты могут быть использованы школьными учителями для развития компетенций учащихся по теме «Комплексные числа», а также преподавателями математики в высших учебных заведениях при выборе методики изучения тем, связанные с комплексными числами.

КЛЮЧЕВЫЕ СЛОВА: преемственность, комплексные числа, алгебра и теория чисел, математический анализ, теория аналитических функций, уровень усвоения, степень абстракции.

\section{ВВЕДЕНИЕ}

Развитие информационного общества ставит свои требования к системе образования. Эти требования относятся какк содержанию образования, так и к результатам обучения. Развитиеинформационных технологий, педагогических идей, результаты международных исследований по образованию (TIIMS, РISAи др.), важность развития STEAMтехнологий обуславливают изменение содержания школьного математического образования. В связи с этим в программу школьной математики Республики Узбекистан были введены такие новые разделы, как комбинаторика, элементы математической логики, комплексные числа, элементы теории вероятности, элементы математической статистики, и финансовой математики.В результате некоторые темы или их содержание, в частности, тема «Комплексные числа», повторяются. Возникает вопрос: «Нужно ли повторное изучение в вузе?» Преподаватели вузов отвечают «Да», так как знания и умения вчерашних учеников по данной тематике не удовлетворяют требованиям вузовской программы. Естественно, возникают вопросы разработки методики изучения темы «Комплексные числа» в школе и в вузе. Поэтому обеспечение преемственности обучения математики в системе «школа-ВУз» вновь становится актуальной. 
Теоретические основы исследования. В научных источниках, в которых рассматривались вопросы преемственности обучения математике в системах «школа-ВУз», «лицей-ВУз» (Turgunbaev, 2012; Алламбергенов, 2019; Антонова, 2005) в основном рассмотрены изучение основ математического анализа.В работе (Мордкович, 2002) для обеспечения преемственности изучения основных понятий анализа предлагают посредством наглядно-интуитивного введения основных понятий алгебры и основ анализа в школе и педагогических вузах. А так как комплексные числа в практику общеобразовательной школы введены недавно, то преемственность изучения этой темы в системе “школаВУз"не рассмотрены.Для достижения целей нашего исследования были рассмотрены работы Р.М.Тургунбаева и И.Алламбергенова (Turgunbaev, 2012; Алламбергенов, 2019; Тургунбаев \& Алламбергенов, 2011; Тургунбаев \& Алламбергенов, 2013). В этих исследованияхв целях обеспечение преемственности изучения основных понятий математического анализа проанализированы повторяющиеся темы в программах математики академических лицеев и математического анализа университетов, изложениеэтих тем в учебной литературе и составлены таблицы учебных элементов, характеризующие уровень усвоения учебных элементов $(\alpha)$, ступеней абстракции, характеризующих язык изложения учебной информации ( $\beta$ ), предложенные В.П.Беспалько (Беспалько, 1989). Таким образом, Р.М.Тургунбаев и И.Алламбергенов предложили свой вариант обеспечения преемственности изучения повторяющихся тем в академическом лицее и университете.

Следует отметить, что в новых школьных программах по математике качество усвоения элементов обучения не указано. Например, в разделе «Комплексные числа» программы по математики указано, что «учащиеся...могут вычислять значения простых выражений с комплексными числами» (Государственный образовательный стандарт, 2017). Но нет никакой информации о сложности этих выражений. Согласно утвержденной новой программе в курсе математики планируется преподавать тему «Комплексные числа». Эти же темы изучаются в высшем учебном заведении, в частности в курсе «Алгебра и теория чисел» направления бакалавриата «5110100-методика преподавания математики». Кроме того, комплексные числа используются в математическом анализе (аналитических функций). Если анализировать учебные пособия, рекомендованные для педагогических вузов (Dixon, 2010; Куликов, 1979; Назаров, 1993; Сирожиддинов, 1978), то видно, что понятия комплексных чисел, арифметика комплексных чисел, геометрическая интерпретация повторяются. Естественно возникает вопрос, надо ли повторное изучение этих материалов, если да, то почему и как. Чтобы ответить на этот вопрос, необходимо ответить на вопросы о том, каковы знания студентов-первокурсников по теме «Комплексные числа». Для того чтобы ответить на эти вопросы, необходимо проанализировать теоретический материал и вопросы, связанные с комплексными числами.Атакже провести контрольную работу со студентами 1 курса, поступившими на математические направления, проанализировать полученные результаты. Важно также уточнить степень усвоения студентами материала, связанного с комплекснымичислами, путем уточнения контекстов преподавания дисциплин «Математический анализ» и «Алгебра и теория чисел».

\section{МАТЕРИАЛЫ И МЕТОДЫ}

В ходе исследования были применены различные методы исследования, а именно: изучение и анализ научнопедагогической, методической, математической литературы и школьных учебников по математике; анализ преемственных связей раздела «Комплексные числа»; педагогический эксперимент с целью проверки остаточных знании учащихся по теме «Комплексные числа», в котором участвовали 89 студентов первого курса Ферганского государственного университета; обсуждение материалов исследования.

\section{РЕЗУЛЬТАТЫ ИССЛЕДОВАНИЯ}

Сначала рассмотрим изложение темы «Комплексные числа» в школьном учебнике (Мирзахмедов, 2017). 4-глава школьного учебника называется «Комплексные числа» и для изучения отводится 6 уроков в конце четвертой четверти 10класса.

Первые два урока посвящены темам «Комплексные числа и действиянад ними» и«Изображения комплексного числа». В данных темах вводится понятие комплексного числа, и связанные с ним понятия: мнимая единица, действительная часть, мнимая часть комплексных чисел, равенство комплексных чисел, арифметические операции над комплексными числами, противоположные комплексные числа, сопряженные комплексные числа, взаимно обратные комплексные числа; вводятся обозначения для комплексных чисел, множества комплексных чисел, мнимой единицы, действительной и мнимой частей, сопряженного комплексного числа, понятие изображение комплексных чисел на координатной плоскости (комплексное число - точка плоскости, комплексное число -радиус-вектор), понятия мнимой и действительной оси, геометрическая интерпретация сложения комплексных чисел - правило параллелограмма.

В этих темах рассматриваются следующие типы упражнений и задач: назовите действительные и мнимые части комплексных чисел; напишите комплексные числа в алгебраической форме, когда заданы действительные и мнимые части; укажите равные комплексные числа; найдитесопряженное числок заданному комплексному числу; найдите сумму, разность, произведение и (иди) частное комплексных чисел; изобразите комплексные числа в плоскости.

На 3-уроке дается информация о комплексных числах в тригонометрической и показательной формах, в частности модуль комплексного числа, аргумент комплексного числа; области значений модуля и аргумента; формы записи тригонометрической и показательной формы комплексного числа (следует отметить, что формула Эйлера не вводится).

Предлагаются упражнения и задачи следующих типов: найдите модуль комплексного числа;найдите аргумент комплексного числа; запишите комплексное число в тригонометрической и показательной формах.

Следующие два урока касаются нахождения действий над комплексными числами, заданными в тригонометрической форме. В данной теме без доказательства приводятся формулы произведения и частного комплексных чисел, заданных в тригонометрической форме, возведения в натуральную степень комплексного числа (формула Муавра). Рассматриваются упражнения и задачи на умножение, деление, возведения в степень 
комплексныхчисел, заданных в тригонометрической форме; на выполнение действий, в которых требуется использование тригонометрические формы комплексных чисел.

Следующий урок посвящентеме извлечения квадратного корня из комплексного числа. Доказывается формула извлечения квадратногокорня их комплексного числа, заданного в тригонометрической форме. При этом формулы извлечения корнейв степени 3 и 4 даются без доказательства. Приведены упражнения и задачинаизвлечения квадратного корня из комплексного числа.

Глава 4 содержит 8 видов упражнений (всего 55 упражнений). Все они могут быть использованы при организации математической деятельности учеников.

4-й модуль учебной программы дисциплины «Алгебра и теория чисел» направления 5110100 - методика преподавания математики (Учебная программа Алгебра и теория чисел, 2018) называется «Алгебраические системы». Его 11-яи 12-темы посвящены изучению поля комплексных чисел, модуля и аргумента комплексного числа и их свойствам; рассматривается геометрический смысл комплексного числа, тригонометрическая форма комплексных чисел, формула Муавра, извлечения корня n-ной степени из единицы и произвольного комплексного числа, аксиоматическая теория комплексных чисел. Для изучения этих тем отведены 8 часов лекций, 8 часов практических занятий и 12 часов самостоятельной работы.

16-й модуль учебной программы дисциплины «Математический анализ» направления5110100 - методика преподавания математики (Учебная программа дисциплины Математический анализ, 2018) называется «Теория аналитических функций». В рамках темы «Комплексная плоскость» планируется изучение понятия «множество комплексных чисел» и установление изоморфизма этого множества с точками евклидовой плоскости, а также понятия плоской линии и области в комплексной плоскости.

Анализ учебников, используемых дляизучения комплексных чисел в школе и ВУЗе показывают, что комплексные числа преподаются в школьной математике с точки зрения расширения понятия чисел, изложение тем изадачный материал направлены на формирование процедурных знаний школьников. В курсе «Алгебра и теория чисел» комплексные числа изучаются как числовая система - математической основой будущей теории полиномов и теории аналитических функций. Множество комплексных чисел является полем, далееизучаются полиномы над полем комплексных чисел. Курс математического анализа рассматривает комплексные числа как числовую систему. Изучается их геометрическая интерпретация, вводится понятие комплексной плоскости, непрерывные кривые и области в комплексной области, изучаются их топологические свойства, аналитические функции и их свойства. Отметим, что в этом курсе доказываются основная теорема алгебры, формула Эйлера, а также даётсяокончательное решение вопроса о степени комплексного числа - определяется произвольная степень произвольного ненулевого комплексного (в частности, действительного) числа.

Очевидно, что изучение комплексного числа как объекта алгебраической системы должно основываться на знании учащихся о комплексных числах, полученных в школьном курсе математики.В то же время,знания, полученные в курсе «Алгебра и теория чисел»,будут использоваться и дополняться в разделе аналитические функции курса математического анализа.

Для оценки остаточных знаний студентов первокурсников по теме «Комплексныкчисла», полученных ими в школьном курсе, в профессиональных колледжах илицеях, было проведено экспериментальное исследование. Онопроводилось в октябре 2019 года в Ферганском Государственном Университете. В эксперименте участвовали студенты первого курса. Студентам были предложенызадачи, подобные тем, которые рассматривались в школьномкурсе математики. Ниже приведен один из вариантов письменной работы.

№1.Укажите равные комплексные числа: 1) $2-4 i$; 2) $2+3 i$;3) $\frac{2}{3}+i$; 4) $\left.\left.\sqrt{121}-7 i ; 5\right) 33+44 i ; 6\right) \sqrt[3]{8}+\sqrt[3]{27} i$;

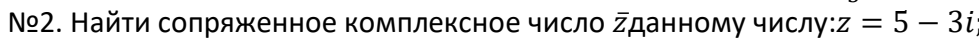

№3. Найти сумму: $(-5+3 i)+(2-i)$;

№4. Найти разность:(3+4i) - $(4+2 i)$;

№5. Найти произведение: $(4+6 i) \cdot(3+4 i)$;

№6. Найти частное: $\frac{2+2 i}{1-2 i}$;

№7. Выполните действия: $\frac{(3-4 i)(4-3 i)}{2+i}$;

№8. Изобразите комплексное число $z=3+4 i$ на плоскости. $z=3+4 i$;

№9. Найти модуль комплексного числа: $z=1+\sqrt{3} i$;

№10. Найти аргумент комплексного числа: $z=\frac{\sqrt{3}}{2}+\frac{1}{2} i$;

№11. Записать в тригонометрической и показательной формах комплексное число: $z=\sqrt{2}-\sqrt{2} i$;

№12.Найтипроизведение: $z_{1}=-\frac{\sqrt{3}}{2}\left(\cos \frac{\pi}{4}+i \sin \frac{\pi}{4}\right) n z_{2}=\frac{1}{2}\left(\cos \frac{\pi}{6}+i \sin \frac{\pi}{6}\right)$;

№13.Найти частное: $z_{1}=\sqrt{2}\left(\cos \frac{\pi}{8}+i \sin \frac{\pi}{8}\right) n z_{2}=2\left(\cos \frac{\pi}{12}+i \sin \frac{\pi}{12}\right)$;

№14. Возвести в степень: $\left(3 \cdot\left(\cos \frac{\pi}{15}+i \sin \frac{\pi}{15}\right)\right)^{5}$;

№15. Извлечь квадратный корень из комплексного числа: $25\left(\cos \frac{\pi}{3}+i \sin \frac{\pi}{3}\right)$;

№16. Записать в алгебраическойформу: $z=\left(\frac{1-\sqrt{3} i}{3 i}\right)^{2}$;

№17.Найти частное: $5\left(\cos 100^{\circ}+i \sin 100^{\circ}\right):\left(\frac{\sqrt{3}}{2}+\frac{1}{2} i\right)$;

№18. Возвести в степень: $\left(\frac{1}{\sqrt{3}}+\frac{1}{\sqrt{3}} i\right)^{10}$; 
№19. Извлечь квадратный корень из комплексного числа: $\sqrt{-27 i}$;

№20. Извлечь кубический корень из комплексного числа: $\sqrt[3]{1+i}$;

№21. Извлечь корень четвертой степенииз комплексного числа: $\sqrt[4]{16}$.

Результаты писменной работы отраженыв таблице 1. Здесь впервой строке даются номера задач, в следующих строках- результаты студентов, закончившихакадемический лицей(АЛ), школу (Ш),профессиональный колледж(ПК). В отдельных строках показаны результаты студентов, окончившие обучениев 2019 году.В скобках указано количество студентов.

Таблица 1.

Результаты контрольной работы

\begin{tabular}{|l|l|l|l|l|l|l|l|l|l|l|}
\hline № задач & 1 & 2 & 3 & 4 & 5 & 6 & 7 & 8 & 9 & 10 \\
\hline АЛ (20) в \% & 70 & 50 & 75 & 80 & 85 & 60 & 50 & 45 & 50 & 5,0 \\
\hline Ш (26) в \% & 73,1 & 53,9 & 92,3 & 92,3 & 84,6 & 65,4 & 57,7 & 46,2 & 61,5 & 7,7 \\
\hline ПК (43) в \% & 72,1 & 46,5 & 93 & 95,4 & 81,4 & 62,8 & 48,8 & 48,8 & 37,2 & 7 \\
\hline 2019(Ш 26)в \% & 73,1 & 53,9 & 92,3 & 92,3 & 84,6 & 65,4 & 57,7 & 46,2 & 61,5 & 7,7 \\
\hline 2019(ПК27)в \% & 66,7 & 55,6 & 92,6 & 92,6 & 77,8 & 59,3 & 51,9 & 48,2 & 33,3 & 3,7 \\
\hline 2019(АЛ19) в \% & 68,4 & 52,6 & 73,7 & 79 & 84,2 & 63,2 & 52,6 & 47,4 & 52,6 & 5,3 \\
\hline 2019 (72) в \% & 69,4 & 54,2 & 87,5 & 88,9 & 81,9 & 62,5 & 54,2 & 47,2 & 48,6 & 5,6 \\
\hline всего (89) в \% & 71,9 & 89 & 91 & 91 & 83 & 62,9 & 51,7 & 47,2 & 47,2 & 6,7 \\
\hline
\end{tabular}

\begin{tabular}{|l|l|l|l|l|l|l|l|l|l|l|l|}
\hline № & 11 & 12 & 13 & 14 & 15 & 16 & 17 & 18 & 19 & 20 & 21 \\
\hline АЛ (20) в \% & 25 & 25 & 40 & 40 & 5,0 & 15,0 & 10,0 & 5,0 & 5,0 & 0,0 & 0,0 \\
\hline Ш (26) в \% & 26,9 & 38,5 & 34,6 & 42,3 & 15,4 & 34,6 & 3,9 & 34,6 & 7,7 & 3,9 & 0,0 \\
\hline ПК (43)в \% & 9,3 & 27,9 & 25,6 & 27,9 & 9,3 & 23,3 & 0,0 & 7 & 4,7 & 7 & 0,0 \\
\hline $2019($ Ш26) в \% & 26,9 & 38,5 & 34,6 & 42,3 & 15,4 & 34,6 & 3,9 & 34,6 & 7,7 & 3,9 & 0,0 \\
\hline 2019(ПК27) в \% & 7,4 & 25,9 & 22,2 & 25,9 & 7,4 & 29,6 & 0,0 & 7,4 & 7,4 & 3,7 & 0,0 \\
\hline 2019(АЛ 19) в \% & 26,3 & 26,3 & 42,1 & 42,1 & 5,4 & 10,5 & 10,5 & 5,3 & 5,3 & 0,0 & 0 \\
\hline 2019 (72) в \% & 19,4 & 30,6 & 31,9 & 36,1 & 9,7 & 26,4 & 4,2 & 16,7 & 6,9 & 2,8 & 0 \\
\hline всего (89) в \% & 18 & 30,3 & 31,5 & 35 & 10,1 & 25 & 3 & 14,6 & 5,6 & 4,5 & 0 \\
\hline
\end{tabular}

Из полученных результатов следует, что большинство студентов правильно решили 3, 4, 5-е примеры, аболее 50\% студентов решили правильно примеры 1, 2, 6, 7. Но большинство оставшихся примеров не были должным образом рассмотрены студентами. Это примеры на геометрическое представление комплексного числа, тригонометрическую форму, выполнение действий над комплексными числами в тригонометрической форме.

Следует также отметить, что результаты практически не зависят от того, окончили ли студенты школу, или академический лицей.

\section{ОБСУЖДЕНИЕ}

Для изучения алгебры и теории чисел, аналитических функций важно, чтобы студенты хорошо знали геометрическую интерпретацию комплексного числа, алгебраическую, тригонометрическую, показательную формы комплексного числа, выполнение действий над таким числами в той или иной форме и их применение.

Анализ программ, учебной литературы (Dixon, 2010; Алламбергенов, 2019; Тургунбаев \& Алламбергенов 2011) показывает, что большинство учебных элементов по теме повторяются. Но, как мы указали выше, тождественного повторения нет, контекст обучения другой. В курсе алгебры и теории чисел изучаются свойства множества комплексных чисел, повышается уровень абстракции. Количество понятий, связанных с комплексными числами увеличивается, комплексные числа рассматриваются как числовая система. То же самое можно наблюдать и в ходе анализа тем, посвященных комплексным числам, курса математического анализа.

Исходя из анализа результатов проведенного эксперимента и анализа учебников, учебных пособий, посвященных изучению комплексных чисел мы предложили следующие уровни усвоения учебных элементов $(\alpha)$ и ступеней абстракции, характеризующих язык изложения учебной информации(таблица 2).

Таблица 2.

Уровни усвоения учебных элементов и ступени абстракции изложения темы “Комплексные числа”

\begin{tabular}{|c|c|c|c|c|c|c|c|c|c|}
\hline \multirow[t]{2}{*}{ № } & \multirow[t]{2}{*}{ Учебные элементы } & \multicolumn{4}{|c|}{$\begin{array}{c}\text { Общеобразовательная } \\
\text { школа }\end{array}$} & \multicolumn{4}{|c|}{$\begin{array}{c}\text { Бакалавриат } \\
\text { (направление методика } \\
\text { преподавания математики) }\end{array}$} \\
\hline & & $\alpha_{H}$ & $\alpha_{k}$ & $\beta_{\mathrm{H}}$ & $\beta_{\mathrm{K}}$ & $\alpha_{H}$ & $\alpha_{k}$ & $\beta_{\mathrm{H}}$ & $\beta_{\mathrm{K}}$ \\
\hline 1 & Комплексное число в алгебраической форме & 0 & 1 & 0 & 1 & 1 & 3 & 1 & 4 \\
\hline 2 & Равные комплексные числа & 0 & 2 & 0 & 1 & 2 & 3 & 1 & 4 \\
\hline 3 & Сопряженные комплексные числа & 0 & 2 & 0 & 1 & 2 & 3 & 1 & 4 \\
\hline 4 & Действиянадкомплексными числами & 0 & 1 & 0 & 1 & 1 & 3 & 1 & 4 \\
\hline 5 & Противоположные комплексные числа & 0 & 2 & 0 & 1 & 2 & 3 & 1 & 4 \\
\hline 6 & Взаимно обратные комплексные числа & 0 & 2 & 0 & 1 & 2 & 3 & 1 & 4 \\
\hline 7 & Изображение комплексных чисел на плоскости & 0 & 1 & 0 & 1 & 1 & 3 & 1 & 4 \\
\hline
\end{tabular}




\begin{tabular}{|c|c|c|c|c|c|c|c|c|c|}
\hline \multirow[t]{2}{*}{ № } & \multirow[t]{2}{*}{ Учебные элементы } & \multicolumn{4}{|c|}{$\begin{array}{c}\text { Общеобразовательная } \\
\text { школа }\end{array}$} & \multicolumn{4}{|c|}{$\begin{array}{c}\text { Бакалавриат } \\
\text { (направление методика } \\
\text { преподавания математики) }\end{array}$} \\
\hline & & $\alpha_{H}$ & $\alpha_{k}$ & $\beta_{\mathrm{H}}$ & $\beta_{\mathrm{K}}$ & $\alpha_{H}$ & $\alpha_{k}$ & $\beta_{\mathrm{H}}$ & $\beta_{\mathrm{K}}$ \\
\hline 8 & Действительная ось, мнимая ось & 0 & 1 & 0 & 1 & 1 & 3 & 1 & 3 \\
\hline 9 & $\begin{array}{l}\text { Изображения комплексного числа в виде } \\
\text { вектора }\end{array}$ & 0 & 1 & 0 & 1 & 1 & 2 & 1 & 3 \\
\hline 10 & $\begin{array}{ll}\text { Правило параллелограмма сложения } \\
\text { комплексныхчисел }\end{array}$ & 0 & 1 & 0 & 1 & 1 & 2 & 1 & 3 \\
\hline 11 & $\begin{array}{l}\text { Тригонометрическая } \\
\text { комплексногочисла }\end{array}$ & 0 & 1 & 0 & 1 & 1 & 2 & 1 & 3 \\
\hline 12 & Комплексные числа в экспоненциальном виде & 0 & 1 & 0 & 1 & 1 & 2 & 1 & 2 \\
\hline 13 & Модуль комплексногочисла & 0 & 1 & 0 & 1 & 1 & 2 & 1 & 4 \\
\hline 14 & Аргументкомплексного числа & 0 & 1 & 0 & 1 & 1 & 2 & 1 & 2 \\
\hline 15 & $\begin{array}{l}\text { Переход от тригонометрического вида к } \\
\text { алгебраическому }\end{array}$ & 0 & 1 & 0 & 1 & 1 & 3 & 1 & 2 \\
\hline 16 & $\begin{array}{l}\text { Умножение и деление комплексных чисел, } \\
\text { заданных в тригонометрической форме }\end{array}$ & 0 & 1 & 0 & 1 & 1 & 3 & 1 & 3 \\
\hline 17 & Формула Муавра & 0 & 1 & 0 & 1 & 1 & 3 & 1 & 3 \\
\hline 18 & Квадратные корни из комплексных чисел & 0 & 1 & 0 & 1 & 1 & 3 & 1 & 3 \\
\hline
\end{tabular}

\section{ВЫВОДЫ И ПЕРСПЕКТИВЫ ДАЛЬНЕЙШЕГО ИССЛЕДОВАНИЯ}

Результаты исследования, в частности приведенные выше таблицы, обсуждались на семинаре кафедры математики и были одобрены преподавателями дисциплин «Математический анализ» и«Алгебра и теория чисел».

Эти результаты могут быть использованы школьными учителями для формирования и развития компетенций учащихся по теме комплексных чисел, а также преподавателями математики в высших учебных заведениях при выборе методов обучения, касающихся данной тематики.

В педагогическом вузе при изучении комплексных чисел такженеобходимо установление преемственных связей между курсами «Математический анализ» (теория аналитических функций) и “Алгебра и теория чисел». Важно ознакомить студентов (будущих учителей математики) с приложениями комплексных чисел к решению задач тригонометрии, планиметрии в кружковых занятиях или в рамках курсов по выбору, при написании курсовых и выпускных работ. Кроме этого, в курсе геометрии студентамможно рекомендовать комплексную интерпретацию геометрии Лобачевского, в курсе математического анализа подробно изучить дробно-линейные функции во взаимосвязи с отображениями на плоскости. В результате у студентов обогащаются знания и умения по теме "Комплексные числа», студенты получают возможность в будущем организовать внеурочную деятельность учащихся школы по данной теме.

\section{Список использованных источников}

1. Dixon M.R., Kurdaschenko L.A., Subbotin I.Ya. Algebra and Number theory. An Integrated Approach. New Jersey, 2010.523 p.

2. Turgunbaev R.M. About some approaches of realization of succession in training elements of the mathematical analysis in the system college - pedagogical university. European Applied Sciences, 2012. №1. P. 202-209.

3. Алламбергенов И.Х. Методика обеспечения преемственности при обучении основам математического анализа в системе академический лицей-университет: Автореф. дисс. PhD/ Нукус, 2019. 50 c.

4. Антонова И.В. Реализация принципа преемственности обучения математике в средней и высшей школах: Дис. ... канд. пед. наук. / М., 2005. 197 с.

5. Беспалько В.П. Слагаемые педагогической технологии. Москва: Педагогика, 1989. 192 с.

6. Государственный образовательный стандарт и учебная программа среднего образования. Физика, математика, информатика, биология, география, химия. Ташкент, 2017. 142 с. (на узб.)

7. Куликов Л.Я. Алгебра и теория чисел. М.: Высшая школа, 1979. $558 \mathrm{c}$.

8. Математика 10-класс (2-часть) /Мирзахмедов М.А. и др. Ташкент: O’qituvchi, 2017. 144 c.

9. Мордкович А.Г. Методические проблемы изучения элементов математического анализа в общеобразовательной школе. Математика в школе. М., 2002. № 9. С. 2-12.

10. Назаров Р.Н., Ташпулатов Б.Т., Дусумбетов А.Д. Алгебра и теория чисел, I часть.Ташкент: O‘qituvchi, 1993. (на узб.)

11. Сирожиддинов С., Максудов Ш., Салохиддинов М. Теория функции комплексного переменного. Т.: Укитувчи, 1978. 367 с. (на узб.)

12. Тургунбаев Р.М., Алламбергенов И.Х. Об обеспечении преемственности в обучении математике в академических лицеях и университетах. Вестник КГУ имени Бердаха. Нукус, 2011. №3-4. С. 42-44 (на узб.)

13. Тургунбаев Р.М., Алламбергенов И.Х. О преемственности в обучении элементам математического анализа (на примере академического лицея-университета). Science and Education New Dimension, 2013. V.5. P. 29-35.

14. Учебная программа дисциплины Математический анализ. Ташкент, 2018. 22 с. (на узб.)

15. Учебная программа дисциплины Алгебра и теория чисел. Ташкент, 2018. 24 с. (на узб.)

\section{References}

1. Dixon M.R., Kurdaschenko L.A., Subbotin I. Ya. (2010) Algebra and Number theory. An Integrated Approach. New Jersey. $523 \mathrm{p}$. 
2. Turgunbaev, R.M. (2012) About some approaches of implementation of succession in training elements of the mathematical analysis in the system college - pedagogical university. European Applied Sciences, No. 1. P. 202-209.

3. Allambergenov, I. Kh. (2019) The methodology for ensuring continuity in teaching the basics of mathematical analysis in the academic lyceum-university system. Abstract. Diss. PhD. Nukus. 50 p.

4. Antonova, I.V. (2005) Implementation of the principle of continuity of teaching mathematics in secondary and higher schools: Dis. ... cand. ped sciences. M. 197 p.

5. Bespalko V.P. (1989) Components of educational technology. Moscow: Pedagogy, $192 \mathrm{p}$.

6. State educational standard and curriculum of secondary education. Physics, mathematics, computer science, biology, geography, chemistry. T., 2017. 142 p. [in Uzbek]

7. Kulikov, L.Ya. Algebra and number theory. M. High School. 1979. 558 p.

8. Mathematics 10-grade (2-part). Mirzakhmedov M.A. et al.T., Ukituvchi. 2017. 144 p.

9. Mordkovich, A.G. (2002) Methodological problems of studying the elements of mathematical analysis in a comprehensive school, 2002. No. 9. P. 2-12.

10. Nazarov, R.N., Tashpulatov, B.T., Dusumbetov, A.D. (1993) Algebra and number theory. Tashkent., I - part [in Uzbek]

11. Sirozhiddinov, S., Maksudov, Sh., Salokhiddinov, M. (1978) Theory of the function of a complex variable. T.: Ukituvchi. 367 p. [in Uzbek]

12. Turgunbaev, R.M., Allambergenov, I.Kh. (2011) On ensuring continuity in the teaching of mathematics at academic lyceums and universities. Bulletin of KSU named after Berdakh. Nukus No. 3-4. P. 42-44 [in Uzbek]

13. Turgunbaev, R.M., Allambergenov, I.Kh.(2013) On continuity in teaching elements of mathematical analysis (for example, academic lyceum-university). Science and Educationa New Dimension. Vol. 5.

14. Curriculum discipline Mathematical analysis. T.: 2018. P. 22 [in Uzbek]

15. The curriculum of the discipline Algebra and number theory. T.: 2018. P. 24 [in Uzbek]

\section{THE CONTINUITY OF THE STUDY ON THETOPIC "COMPLEX NUMBERS" IN SECONDARY SCHOOLS AND IN PEDAGOGICAL UNIVERSITIES OF THE REPUBLIC OF UZBEKISTAN Tolkinjon Yunusalievich Bakirov}

Abstract.

Fergana state university, Uzbekistan

Brief descriptions of the problem. In connection with the reforms carried out in the field of education in the Republic of Uzbekistan, the content of secondary education, in particular mathematical education is changing. This article discusses the question of studying succession of the topic "Complex numbers" at school and pedagogical university, and changes in the content of secondary education, in particular mathematical education. In the curriculum of school mathematics of the Republic of Uzbekistan, such new sections as combinatorics, elements of mathematical logic, complex numbers, elements of probability theories, elements of mathematical statistics, and financial mathematics were introduced. As a result, some topics or their content, the particular topic "Complex numbers", are repeated in the course of university mathematics. It is necessary to develop a methodology for studying newly introduced sections of mathematics, especially some recurring topics, to indicate the levels of assimilation of repetitive concepts by pupils of school and students of university.

Materials and methods. During the study, various research methods were applied, namely, the study and analysis of scientific and pedagogical, methodological, mathematical literature and school textbooks in subject of mathematics; analysis of the continual relationships of the section "Complex numbers"; a pedagogical experiment, in which 89(eighty nine) first-year students of Fergana State University were participated, was hold to test the residual knowledge of students on the topic "Complex numbers"; research materials were discussed.

Results. In the course of the study, the levels of understanding of educational elements and levels of abstraction characterizing the language of presentation of educational information on the topic "Complex numbers" were proposed.

Conclusion. The results obtained can be used by schoolteachers to develop the knowledge and competence of students on the topic of complex numbers, as well as by mathematics teachers in higher educational institutions when choosing a methodology for teaching complex numbers.

Keywords: succession, complex numbers, algebra and number theory, mathematical analysis, theory of analytical functions, level of assimilation, degree of abstraction.

\section{$(\mathrm{Cc}) \mathrm{EY}-\mathrm{NC}-\mathrm{SA}$}

This work is licensed under Creative Commons Attribution-NonCommercial-ShareAlike 4.0 International License. 